\title{
Predicting vapor liquid equilibria using density functional theory: A case study of argon
}

Himanshu Goel, Sanliang Ling, Breanna Nicole Ellis, Anna Taconi, Ben Slater, and Neeraj Rai

Citation: The Journal of Chemical Physics 148, 224501 (2018); doi: 10.1063/1.5025726

View online: https://doi.org/10.1063/1.5025726

View Table of Contents: http://aip.scitation.org/toc/jcp/148/22

Published by the American Institute of Physics

\section{Articles you may be interested in}

Perspective: Ab initio force field methods derived from quantum mechanics

The Journal of Chemical Physics 148, 090901 (2018); 10.1063/1.5009551

Stochastic thermodynamics and entropy production of chemical reaction systems

The Journal of Chemical Physics 148, 224104 (2018); 10.1063/1.5037045

Communication: Simple liquids' high-density viscosity

The Journal of Chemical Physics 148, 081101 (2018); 10.1063/1.5022058

Announcement: Top reviewers for The Journal of Chemical Physics 2017

The Journal of Chemical Physics 149, 010201 (2018); 10.1063/1.5043197

Aqueous solvation from the water perspective

The Journal of Chemical Physics 148, 234505 (2018); 10.1063/1.5034225

Crystallization of hard spheres revisited. II. Thermodynamic modeling, nucleation work, and the surface of tension

The Journal of Chemical Physics 148, 224102 (2018); 10.1063/1.5025394

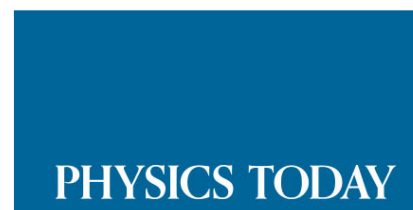

WHITEPAPERS
ADVANCED LIGHT CURE ADHESIVES

Take a closer look at what these environmentally friendly adhesive systems can do

\section{READ NOW}

PRESENTED BY

Q. MASTERBOND' 


\title{
Predicting vapor liquid equilibria using density functional theory: A case study of argon
}

\author{
Himanshu Goel, ${ }^{1}$ Sanliang Ling, ${ }^{2,3}$ Breanna Nicole Ellis, ${ }^{1}$ Anna Taconi, ${ }^{1}$ Ben Slater, ${ }^{2}$ \\ and Neeraj Rai ${ }^{1, a)}$ \\ ${ }^{1}$ Dave C. Swalm School of Chemical Engineering, and Center for Advanced Vehicular Systems, \\ Mississippi State University, Mississippi State, Mississippi 39762, USA \\ ${ }^{2}$ Department of Chemistry, University College London, 20 Gordon Street, London WC1H OAJ, United Kingdom \\ ${ }^{3}$ Advanced Materials Research Group, Faculty of Engineering, University of Nottingham, University Park, \\ Nottingham NG7 2RD, United Kingdom
}

(Received 12 February 2018; accepted 24 May 2018; published online 12 June 2018)

\begin{abstract}
Predicting vapor liquid equilibria (VLE) of molecules governed by weak van der Waals (vdW) interactions using the first principles approach is a significant challenge. Due to the poor scaling of the post Hartree-Fock wave function theory with system size/basis functions, the Kohn-Sham density functional theory (DFT) is preferred for systems with a large number of molecules. However, traditional DFT cannot adequately account for medium to long range correlations which are necessary for modeling vdW interactions. Recent developments in DFT such as dispersion corrected models and nonlocal van der Waals functionals have attempted to address this weakness with a varying degree of success. In this work, we predict the VLE of argon and assess the performance of several density functionals and the second order Møller-Plesset perturbation theory (MP2) by determining critical and structural properties via first principles Monte Carlo simulations. PBE-D3, BLYP-D3, and rVV10 functionals were used to compute vapor liquid coexistence curves, while PBE0-D3, M06-2X-D3, and MP2 were used for computing liquid density at a single state point. The performance of the PBE-D3 functional for VLE is superior to other functionals (BLYP-D3 and $\mathrm{rVV} 10$ ). At $\mathrm{T}=85 \mathrm{~K}$ and $\mathrm{P}=1 \mathrm{bar}$, MP2 performs well for the density and structural features of the first solvation shell in the liquid phase. Published by AIP Publishing. https://doi.org/10.1063/1.5025726
\end{abstract}

\section{INTRODUCTION}

The knowledge of thermophysical properties such as vapor liquid equilibria (VLE) is critical for the design and development of numerous separation processes. The classical empirical potentials are often parametrized against experimental data for computing thermophysical properties at ambient or subcritical state points. ${ }^{1-5}$ The performance of these force fields becomes unreliable when considering properties or state points not included in the parametrization. There is also a significant emphasis on developing an ab initio interatomic potential by using quantum chemical calculations and using them to predict bulk phase properties. ${ }^{6-25}$ The potential energy surface for a large number of configurations is generated to fit to the functional form of the force field. This approach is, however, limited to moderately sized molecules with limited conformational degrees of freedom as the computational cost increases significantly with the complexity of the molecule.

Rapid advances in computational resources and efficient algorithms have contributed immensely to the development and application of electronic structure calculations. This expands the capacity to simulate bigger system size and model complex molecules, by significantly reducing the computational time. Recent density functionals (DF) and

a)Electronic mail: neerajrai@che.msstate.edu post Hartree-Fock (HF) wave function theory can model small molecules with reasonable accuracy. However, modeling condensed phase systems, in particular, multi-phase phenomena such as vapor liquid equilibria, remains a significant challenge. The popular Kohn-Sham density functional theory (KS-DFT) ${ }^{26,27}$ is widely used in material science for modeling condensed phase properties. One of the biggest challenges for KS-DFT is to accurately account for weak non-covalent interactions. ${ }^{28-30}$ Advances in the DFT are largely based on improving the performance of exchangecorrelation $(\mathrm{XC})$ functionals by incorporating additional information of the electronic system. Perdew et al. ${ }^{31}$ explain the construction of XC functionals through "Jacob's ladder" where different rungs were classified based on the density functional approximations to define XC energies. As of now, there are five rungs starting from local density approximation (LDA), ${ }^{32,33}$ generalized gradient approximation (GGA), ${ }^{34-37}$ meta-GGA, ${ }^{38-42}$ hybrid functionals, ${ }^{43-47}$ and random phase approximation (RPA). ${ }^{48-51}$ As one climbs higher steps in the ladder, it leads to greater accuracy albeit at a higher computational cost. Additional details regarding the hierarchy of DF methods can be accessed through the work of Perdew and co-workers. ${ }^{31,52-54}$

Over the last two decades or so, many improvements in DFT have been through the addition of dispersion correction terms to the XC energies. A concept similar to "Jacob's ladder" was introduced by Klimeš and Michaelides ${ }^{29}$ to classify 
different DFT based dispersion correction schemes. Going higher on the ladder leads us to greater accuracy, less empiricism, and higher computational cost. At present, the pair-wise additive dispersion correction models ${ }^{28,55-57}$ and nonlocal van der Waals (vdW) functionals ${ }^{58-61}$ have demonstrated some success in handling weak vdW interactions. These models are usually benchmarked against data sets of dimer energetics, and their performance in estimating bulk phase properties precisely is still a concern.

This work is aimed at obtaining vapor liquid coexistence curves (VLCCs), critical properties, and structural properties for argon via first principles Monte Carlo (FPMC) simulations and assess the performance of several density functionals (GGA, hybrid, and rVV10 nonlocal vdW functionals) along with second order Møller-Plesset perturbation theory (MP2). Being an important noble gas, argon has been studied extensively for establishing benchmarks. Earlier VLE studies with FPMC simulations were performed on water, ${ }^{62-64}$ methane ${ }^{65}$ methanol ${ }^{65}$ hydrofluorocarbons, ${ }^{66,67}$ carbon dioxide, ${ }^{68}$ and sulfur dioxide. ${ }^{68}$ For argon, the work carried out by Maerzke et al. ${ }^{69}$ presents several key results including dimer potential energy curves (PECs) and liquid structure by using self-consistent polarization density functional theory. However, their bulk phase calculation was limited to a single temperature $(85 \mathrm{~K})$. Given the significance of argon as a prototypical system governed by dispersion interactions, it is useful to determine VLE directly from first principles and examine the performance of several density functionals. In the past, most of the studies were performed by using the GGA class of functionals, and the accuracy of higher rung functionals is rarely tested. With the relatively low cost of GGA functionals, they are still very popular and widely used with reasonable accuracy. However, researchers are moving toward hybrid density functionals and electron correlation methods such as RPA, and MP2, to predict bulk phase properties with greater accuracy as compared to GGA functionals. ${ }^{70-84}$

The rest of the paper is organized as follows. Section II describes the details of density functionals, simulation setup, dispersion models and Monte Carlo method. In Sec. III, we present potential energy curves, second virial coefficients, and results obtained from Monte Carlo simulations. The results comprise of VLCCs, Clausius-Clapeyron plots, ${ }^{85}$ critical properties, and structural investigation of the liquid phase argon. At last, concluding remarks are presented in Sec. IV.

\section{COMPUTATIONAL METHODS}

In this work, two sets of simulations were performed. First, we calculate the vapor liquid coexistence curves of argon, which involves two thermodynamically connected simulation boxes for computing saturated liquid and vapor densities at different temperatures. Second, using $N p T$ ensemble, the density is computed at a single temperature. First set of calculations were performed by using the PBE, ${ }^{37}$ BLYP $^{35,36}$ GGA functionals and the $\mathrm{rVV} 10^{86}$ nonlocal van der Waals functional. The GGA functionals particularly PBE and BLYP are well known and were extensively used due to reasonable accuracy and modest computational cost. Nonlocal functionals add a nonlocal correlation energy term to the local or semilocal functional, which results in increased computational cost to some degree but improves performance. We have used the $\mathrm{rVV} 10^{86}$ functional which is a revised version of the VV10 ${ }^{87}$ functional. The rVV10 functional is chosen because it is one of the best-performing nonlocal correlation functionals for accurate predictions of the equilibrium bond length and interaction energy of argon dimer and the equilibrium lattice constant and cohesive energy of solid argon. ${ }^{88}$ The second set or single temperature calculations were carried out using PBE $0,{ }^{45,89}$ $\mathrm{M} 062 \mathrm{X},{ }^{47}$ and the second-order Møller-Plesset perturbation theory (MP2). ${ }^{90-94}$ We could do these calculations only at a single temperature due to extremely high computational cost. In order to consider the long range dispersion interactions, we have used dispersion correction DFT-D $3^{57}$ developed by Grimme and co-workers. The DFT-D3 method is used with PBE, BLYP, PBE0 and M06-2X functionals, respectively. In order to determine the VLCCs for argon, we have used the canonical version of the Gibbs ensemble Monte Carlo (GEMC) method ${ }^{95}$ and the $N p T^{96}$ ensemble was employed to calculate the liquid density at a specified temperature and pressure. The GEMC simulation setup utilizes two separate periodic simulation boxes for representing liquid and vapor phases connected thermodynamically via a unified partition function..$^{97}$ For GEMC simulation, total number of molecules, total volume of both boxes, and temperature of the system are kept constant. The Monte Carlo simulation contains various trial moves to sample the configurational space. These moves include translation and changes in the volume of the simulation box for $N p T$ simulations. In addition, the swap moves between liquid and vapor boxes were also performed for GEMC simulation to equilibrate the chemical potential. The probabilities of volume, swap, and translational move types are $15 \%-20 \%, 20 \%-25 \%$, and 60\%, respectively, and the acceptance rate of the moves ranges from $50 \%$ to $60 \%$. Most of the simulations were carried out using the triple-zeta valence basis set augmented with polarization functions (TZV2P) and Godecker-Teter-Hutter (GTH) pseudopotentials. ${ }^{98,99}$ For dispersion corrected hybrid functionals (PBE0-D3, M06-2X-D3), the auxiliary density matrix method (ADMM) with the pFIT3 auxiliary basis was employed. ${ }^{76}$ The benefit of the ADMM method is to reduce the computational cost by considering a smaller auxiliary basis for nonlocal Hartree-Fock exchange (HFX) calculations. For MP2 simulation, we used the Gaussian and plane wave MP2 approach with the resolution of identity (RI) approximation ${ }^{100,101}$ which was recently implemented in the CP2K code. The triple-zeta quality valence-only correlation consistent type primary basis set and associated auxiliary RI basis set (see the supplementary material) were generated and used for the RI-Gaussian and Plane Wave (GPW)-MP2 calculation. The procedure for generating these basis sets has been discussed in the work of Del Ben et al. ${ }^{101}$ and we refer the interested reader to the original reference for more information. The truncation radius for Coulomb interaction was set to $7 \AA$ for hybrid DFT and MP2 calculations. All computational details such as XC functionals, GTH pseudopotentials, and their plane wave cutoff are explicitly provided in the supplementary material. All MC simulations presented in this 
work have used the $\mathrm{CP} 2 \mathrm{~K}$ software suite (version 2.6.2 and 5.0). ${ }^{102}$ The CP2K software suite uses KS-DFT to compute interaction energies via the Quickstep module ${ }^{103}$ employing a hybrid scheme of the Gaussian and Plane Wave (GPW) method.

First principles MC simulations require large computational resources. This computational cost can be considerably reduced by using approximate bias potentials to sample the configurational space. ${ }^{104-107}$ In this scheme, the configurational space is first sampled by approximate bias potentials for a short sequence of moves followed by the DFT calculation. The energy difference between DFT and the approximate bias potential is used to compute acceptance criteria to either accept or reject the entire (short) sequence of moves. We have used 16 moves for the short sequence using approximate bias potentials. ${ }^{62}$ The nonbonded interaction parameters for the approximate bias potential are taken from Michels' work. ${ }^{108}$ All GEMC/NpT simulations (except MP2) were run for 500-600 MC cycles. Each cycle refers to $N$ (total number of molecules) moves with Quickstep energy calculations. The first 250-300 cycles were considered to be the equilibration, and the remaining cycles were considered for data collection. The production run was divided into blocks of 50 cycles to calculate the average and standard deviations for saturated liquid and vapor densities. For MP2 simulation, we performed four independent runs by using different seed and probability ratios. Each independent simulation consisted of $175 \mathrm{MC}$ cycles. The first $100 \mathrm{MC}$ cycles were considered as equilibration and the remaining 75 cycles from each independent run were used to calculate the average density and corresponding standard deviation. The approximate bias parameters and the numerical values for VLCCs can be found in the supplementary material. The critical temperature and critical density of argon for different functionals are also calculated by using subcritical VLCC data. For this purpose, the density scaling law with critical exponent 0.325 and the law of rectilinear diameter were used. ${ }^{109-111}$ Additionally, the normal boiling point was calculated by fitting vapor pressure data to the Clausius-Clapeyron equation.

We performed GEMC calculations with the PBE-D3 functional in order to choose an appropriate system size (number of molecules) for this study. The system sizes considered are $36,54,64,72,128$, and 256 argon atoms. Figure 1 shows

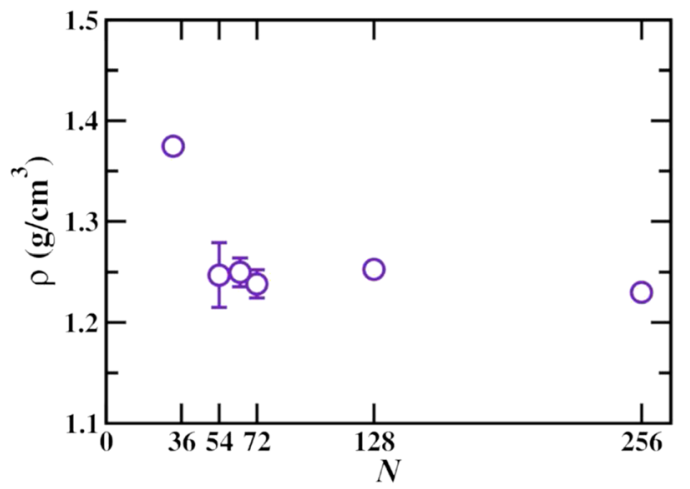

FIG. 1. Effect of system size $(N)$ on the liquid density of argon at $85 \mathrm{~K}$ and 1 bar. The error bars smaller than the symbol size are not shown. the plot for the saturated liquid density of argon as a function of system size. It is evident from the figure that the density converges to the large system size limit after 54 particles. There is a somewhat larger standard deviation for the density obtained from 54-particle simulation. The density obtained from the system size of 64 or 72 argon atoms is reliable and appears suitable for the simulation study. Thus, we have used 72 argon atoms for the GGA and hybrid functional calculations (PBE0-D3 and M06-2X-D3) and 64 particles for MP2 simulations. We have used a smaller system size for MP2 simulations because of the significant computational cost associated with the larger simulation cell. We note that the MP2 method has recently been applied to study liquid water, and excellent agreements have been obtained with the experiment on water density and radial distribution functions (RDF). ${ }^{71}$ The initial system setup procedure can be referred from our previous work. ${ }^{66}$ The structural analysis for the liquid phase of argon was determined through radial distribution function (RDF) plots.

As the two-body interaction term contributes most to the total potential energy of the $n$-body system, it can be helpful to assess the performance of various functionals for dimer energetics. The potential energy curves (PECs) can provide an insight into the accuracy of bulk phase property prediction. The argon PECs through different functionals are compared with coupled cluster single, double, and perturbative triple excitations $(\operatorname{CCSD}(\mathrm{T}))^{112-117}$ level of theory with extrapolation to the complete basis set (CBS) limit. ${ }^{7}$ Most of the GPW dimer calculations and VLCCs in this work have been performed using the TZV2P basis set, which was found to converge well for the equilibrium bond length and interaction energy of the argon dimer. Therefore, it is also beneficial to obtain the PECs from the CCSD(T) using the triple zeta basis set. For this purpose, we carried out the argon dimer calculation to obtain the PECs from the $\operatorname{CCSD}(\mathrm{T}) /$ aug-ccpVTZ(aVTZ) basis set. ${ }^{118,119}$ We have used the Boys and Bernardi counterpoise correction ${ }^{120}$ for removing the basis set superposition error. The CCSD(T)/aVTZ calculations for the potential energy curve were performed by using the Gaussian 09 software. ${ }^{121}$

The potential energy curve between two argon atoms can be used to compute the second virial coefficient using the following equation:

$$
B_{2}(T)=-2 \pi \int_{0}^{\infty}\left(e^{-U(r) /\left(k_{\mathrm{B}} T\right)}-1\right) r^{2} d r
$$

where $U(r), k_{B}$, and $T$ represent the dimer potential at separation $r$, the Boltzmann constant, and temperature, respectively. To compute $B_{2}(T)$, we have followed the approach used by Maerzke et al. ${ }^{69}$ However, we did not include the quantum corrections to the virial coefficients as they appear to be less than $1 \% .{ }^{69}$ In brief, $B_{2}(T)$ was determined by using trapezoidal numerical integration in the range of $r=0-50.0 \AA$. The energies were set to a large positive number in the region of $r=0-2.0 \AA$, which results in the Mayer function to be -1 . For every functional, we have computed the dimer potential energy for $r=2.0-7.0 \AA$, and the local cubic splines were used to interpolate the energies in this region. The potential 
energies obtained over the region $r=6.25-7.0 \AA$ were used to fit the functional form $-C_{6} r^{-6}$. Thereafter, the value for $C_{6}$ coefficient was used to extrapolate the potential energies from 7.0 to $50.0 \AA$.

\section{RESULTS AND DISCUSSION}

\section{A. Dimer energetics}

The leading contribution to the total energy for an $n$-body system is from two-body terms. Thus, dimer potential energy curves (PECs) can be a good indicator for the performance of different levels of theory for predicting condensed phase thermodynamic properties. Figure 2 shows the PECs of argon with different classes of functionals and MP2. The location of the minima and the interaction energy are also listed in Table I. The curve obtained from the CCSD(T)/aug-cc-pVTZ(aVTZ) basis set overestimates the equilibrium bond length and underestimates the binding energy when compared with $\mathrm{CCSD}(\mathrm{T}) / \mathrm{CBS}$ or the experiment.

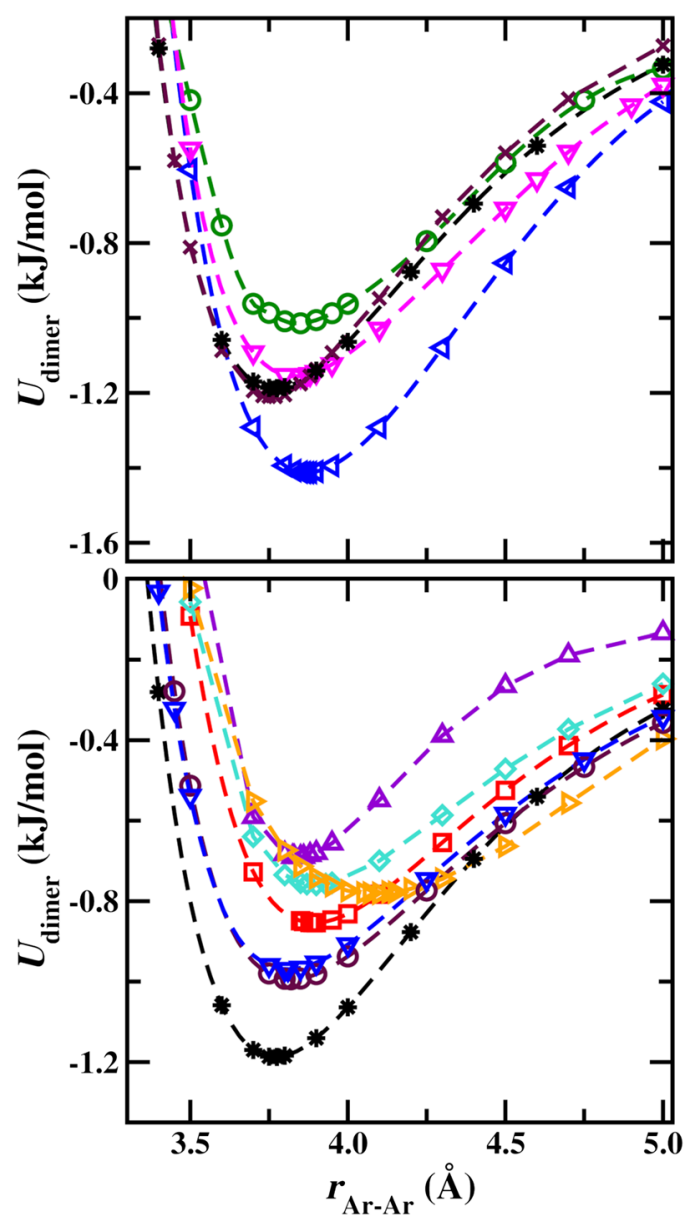

FIG. 2. Potential energy curves of argon dimer at different levels of theory. The black stars, red squares, violet triangle up, blue triangle left, magenta triangle down, maroon cross, orange triangle right, turquoise diamonds, green circles, maroon circles, and blue triangle down represent data for CCSD(T)/CBS, ${ }^{7}$ CCSD(T)/aVTZ (aug-cc-pVTZ), BLYP-D3/GPW, PBED3/GPW, PBE0-D3/GPW, rVV10/GPW, M06-2X-D3/GPW, MP2/GPW, MP2/aVTZ, LJ (Michels et al.), ${ }^{108}$ and LJ (Vrabec et al. $)^{23}$ potentials, respectively. The dashed lines of the corresponding color are guide to the eye.
TABLE I. The location of well depth $\left(R_{0}, \AA\right)$ and interaction energy $\left(E_{\mathrm{int}}\right.$, $\mathrm{kJ} / \mathrm{mol}$ ) of argon at the minima in the dimer potential energy curve for different methods compared with $\operatorname{CCSD}(\mathrm{T})$ and experimental work.

\begin{tabular}{lcc}
\hline \hline Functional & $R_{0}$ & $-E_{\text {int }}$ \\
\hline PBE-D3/GPW & 3.87 & 1.412 \\
BLYP-D3/GPW & 3.85 & 0.690 \\
rVV10/GPW & 3.77 & 1.210 \\
PBE0-D3/GPW & 3.85 & 1.155 \\
M062X-D3/GPW & 4.10 & 0.780 \\
MP2/GPW & 3.90 & 0.759 \\
MP2/aVTZ & 3.85 & 1.014 \\
LJ (Vrabec et al. $^{23}$ ) & 3.81 & 0.969 \\
${\text { LJ }\left(\text { Michels } \text { et al. }^{108} \text { ) }\right.}_{\text {CCSD(T)/aVTZ }}$ & 3.82 & 0.994 \\
CCSD(T)/CBS & 3.89 & 0.853 \\
Experiment $^{122}$ & 3.767 & 1.187 \\
\hline
\end{tabular}

aug-cc-pVTZ (aVTZ)

Complete basis set (CBS)

Gaussian plane wave (GPW)

For GGA functionals (PBE and BLYP with dispersion correction D3), the equilibrium bond lengths from both functionals are overestimated by approximately $0.1 \AA$ in comparison with the experiment. The binding energy is largely underestimated for BLYP-D3 and overestimated by the PBED3 functional compared to the experimental values. This binding energy difference for the BLYP-D3/PBE-D3 functionals can have significant consequences on the nature of VLCCs (see below). In the case with hybrid functionals, the PBE0-D3 functional overestimated the bond length marginally with a difference of $0.09 \AA$ as compared to the experiment or CCSD(T)/CBS. The PBE0-D3 functional binding energy at the equilibrium distance is close enough to the experiment or $\operatorname{CCSD}(\mathrm{T}) / \mathrm{CBS}$, but it fails to carry the similar performance in the long range interaction region where it is more attractive than CCSD(T)/CBS. The M06-2X functional is based on hybrid meta-exchange-correlation functionals and accounts for short to medium range correlation. The M062X-D3 functional completely fails to determine the equilibrium bond length and it overestimated the value by $0.40 \AA$. Furthermore, the binding energy obtained from the M062X-D3 functional underestimates the experimental well depth.

For the nonlocal functional rVV10, we can see that the calculated equilibrium bond length and the binding energy are in good agreement with the experimental data. The PEC obtained from the rVV10 functional matches well with the CCSD(T)/CBS curve until $4 \AA$. However, the rVV10 PEC does not provide similar accuracy as the distance between particles increases beyond $4 \AA$. After $4 \AA$, the interaction energy is slightly underestimated when compared to the CCSD(T)/CBS curve. In addition, the argon PEC with the rVV10 functional is in good agreement with the reference curve as shown in the work of Sabatini et al. ${ }^{86}$ and Tran and Hutter. ${ }^{88}$ Next, the MP2 method well known for accounting electron correlation is computationally efficient as compared to coupled cluster and configuration interaction methods. The results obtained from 
the MP2/GPW and MP2/aVTZ show the binding energy difference of around $0.25 \mathrm{~kJ} / \mathrm{mol}$. Compared to the experimental data, both underestimate the binding energy and overestimate the equilibrium bond length. In summary, the rVV10 functional is the best functional for dimer energetics, which is consistent with our earlier published studies on hydrofluorocarbons as well. ${ }^{66,68}$ According to the work of Tran and Hutter ${ }^{88}$ the rVV10 functional also performs well as compared to other tested DFT functionals in case of rare gas dimers. After the rVV10 functional, the PBE0-D3 functional also does a decent job for the equilibrium bond length and binding energy as compared to the experiment/CCSD(T)/CBS. The effective $\mathrm{LJ}$ pair potentials developed by Michels et al. ${ }^{108}$ and Vrabec et $a l^{23}$ underestimate the potential well depth by approximately $16 \%$ and $18 \%$, respectively. Since the LJ potential by Vrabec et al. ${ }^{23}$ can provide an extremely good prediction for the vapor liquid coexistence curve, it suggests that many-body polarization effects are repulsive in nature.

\section{B. Second virial coefficients}

The second virial coefficients for argon with different functionals and LJ potential ${ }^{23}$ are shown in Fig. 3 and compared with the experimental ${ }^{123}$ and $\operatorname{CCSD}(\mathrm{T}) / \mathrm{CBS}^{7}$ data over the range of $100-1000 \mathrm{~K}$. The numerical values for second virial coefficients are also provided in Table S6 of the supplementary material. Among all the functionals and the LJ potential $^{23}$ used here, the best performance is shown by the PBE0-D3 and rVV10 nonlocal functional. At lower temperatures $(100-200 \mathrm{~K})$, the unsigned mean percentage errors are $7.5 \%$ and $8.0 \%$ for PBE0-D3 and rVV10 functionals as compared to the experimental data. Moreover, the predictions for second virial coefficients at higher temperatures are also in good agreement with experimental data. The next closest results are provided by the LJ potential. ${ }^{23}$ The PBE-D3 functional significantly overestimates, while BLYP-D3, MP2, and M06-2X-D3 significantly underestimate $B_{2}(T)$.

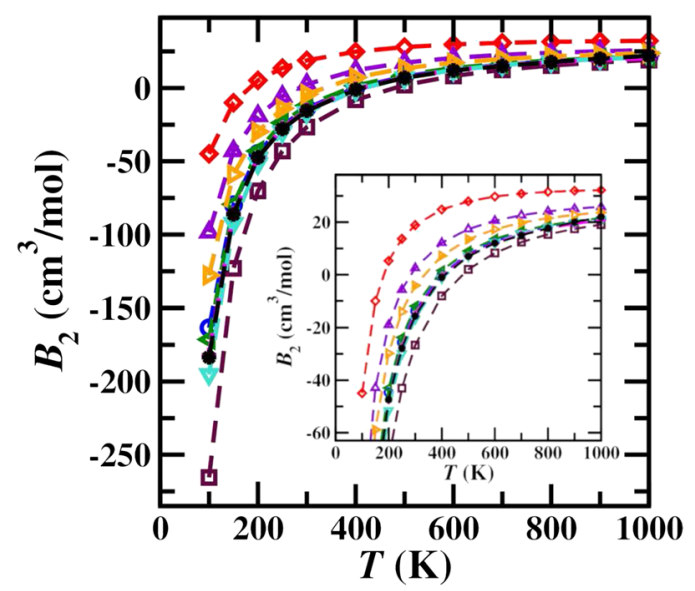

FIG. 3. Temperature dependence of the second virial coefficient of argon. The black stars, magenta pluses, red diamonds, maroon squares, turquoise triangle down, green triangle left, orange triangle right, violet triangle up, and blue circles represent data for the experiment, ${ }^{123} \mathrm{CCSD}(\mathrm{T}) / \mathrm{CBS},{ }^{7}$ BLYPD3/GPW, PBE-D3/GPW, PBE0-D3/GPW, rVV10/GPW, M06-2X-D3/GPW, MP2/GPW, and LJ (Vrabec et al. $)^{23}$ potential, respectively. The dashed lines of the corresponding color are a guide to the eye.

\section{Liquid density and structure}

The performance of GGA (PBE-D3 and BLYP-D3), rVV10, hybrid (PBE0-D3, M06-2X-D3), and MP2 methods is compared by predicting liquid densities for argon at $85 \mathrm{~K}$ and 1 bar. Table II lists down the computed densities for different functionals and MP2. The accuracy of the functionals in predicting density is in this order: MP2 > M06-2X-D3 $>$ PBE0-D3 > BLYP-D3 > PBE-D3 > rVV10. Clearly, the MP2 method provides the best estimate of density with a $2.2 \%$ error. From the $B_{2}(T)$ values for MP2, one could not have come to the conclusion that MP2 would be able to provide such a good estimate for the liquid density. It appears as the system size increases, MP2 overestimates many-body polarization effects, leading to the cancellation of errors. The only constraint in doing MP2 calculations for the condensed phase is the need for substantial computational resources. The second best estimate comes from the M06-2X-D3 functional with an underprediction of $2.3 \%$. We could also test the accuracy of the M06-2X-D3 functional at a slightly higher temperature $(105 \mathrm{~K})$ and the computed density is underpredicted by approximately $6.5 \%$. The M06-2X-D3 does a decent job in computing the density in spite of poor performance for the well depth location of argon dimer. The performance of another hybrid functional PBE0-D3 is somewhat reasonable for the density and PEC of argon. The computed density is overpredicted by 8.4\%. The density obtained from BLYP-D3 and PBE-D3 functionals are underestimated by $10 \%$ and $12 \%$, respectively. At $85 \mathrm{~K}$, both functionals show similar accuracy for liquid density in spite of the large difference in the binding energy, suggesting that at lower temperatures the location of the minima in PEC is more important than the binding energy. As described above, the difference in binding energy mainly affects the bulk phase properties at higher reduced temperatures.

The structural features of the liquid phase of argon are explored through analyzing the radial distribution function. Figure 4 shows the RDF plots with different functionals at $85 \mathrm{~K}$ and their characteristics (location of the first coordination shell and height of the peak) are provided in Table III. The experimental RDF is taken from the work of Yarnell et al. ${ }^{125}$ Except for PBE-D3 and BLYP-D3, most of the other functionals perform reasonably well in predicting the location of the first coordination shell. The PBE-D3 and BLYP-D3 functionals somewhat overestimate the location of the peak, 3.86 and $3.74 \AA$, respectively, as compared to the experimental

TABLE II. Liquid density of argon at $T=85 \mathrm{~K}$ and $P=1$ bar with different methods and LJ potential compared with experiment. Experimental data are taken from the NIST chemistry webbook. ${ }^{124}$

\begin{tabular}{lccc}
\hline \hline Functional & $\rho\left(\mathrm{g} / \mathrm{cm}^{3}\right)$ & St. dev. & \% error \\
\hline PBE-D3 (at 85 K) & 1.238 & 0.014 & -12.14 \\
BLYP-D3 (at 85 K) & 1.268 & 0.016 & -10.0 \\
rVV10 (at 85 K) & 1.621 & 0.006 & 15.04 \\
PBE0-D3 (at 85 K) & 1.527 & 0.010 & 8.37 \\
M062X-D3 (at 85 K) & 1.377 & 0.016 & -2.27 \\
MP2 (at 85 K) & 1.440 & 0.014 & 2.20 \\
LJ (Vrabec et al. $\left.{ }^{23}\right)$ & 1.407 & 0.002 & -0.15 \\
Expt. (at 85 K) & 1.409 & & \\
\hline \hline
\end{tabular}




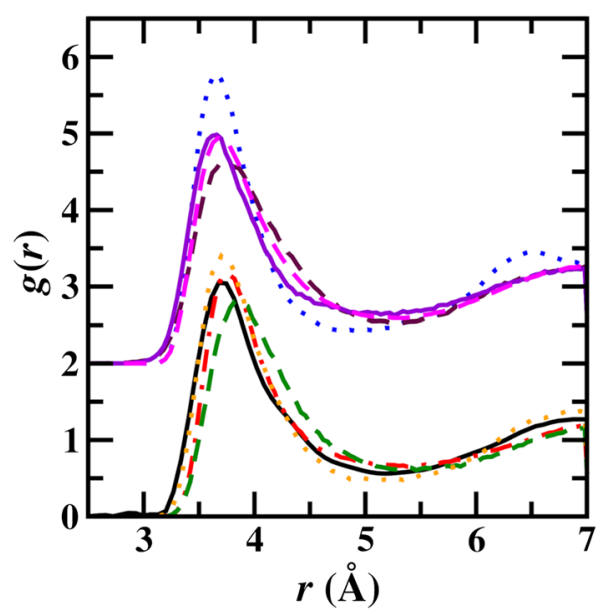

FIG. 4. Liquid phase radial distribution functions for argon at $85 \mathrm{~K}$. The dash-dotted red, dashed green, and dotted orange color lines represent results obtained with BLYP-D3, PBE-D3, and PBE0-D3, respectively. The solid violet, dashed maroon, dotted blue, and dashed magenta color lines represent results obtained with MP2, M062X-D3, rVV10, and LJ (Vrabec et al. ${ }^{23}$ ) potentials, respectively (an offset of +2 was added to the $y$-coordinates). The solid black line denotes the experimental data taken from the work of Yarnell et al. ${ }^{125} \mathrm{~A}$ bin width of $0.04 \AA$ is used for all RDF plots.

value of $3.68 \AA$. This is also consistent with the fact that liquid density is underestimated for these functionals. The peak height with PBE0-D3 and rVV10 functionals is considerably higher as compared to other functionals and this is largely due to higher densities predicted with both of these functionals. The peak height obtained from the M062X-D3 functional is slightly smaller despite a reasonable density prediction. The liquid structure obtained from MP2 based MC simulations is in excellent agreement with experimental data. This is somewhat expected since the density obtained with MP2 energetics is also very close to the experimental liquid density. The location and the height of the first solvation peak for the LJ pair potential is also in good agreement with the experimental data (see Table III).

\section{Vapor liquid phase equilibria}

The VLCCs for argon obtained from GEMC simulations are shown in Fig. 5. This figure compares the performance of three different functionals (PBE-D3, BLYP-D3, and rVV10) against the experimental data. The overall saturated liquid densities obtained from PBE-D3 and BLYP-D3 functionals are underestimated by $8.5 \%$ and $14 \%$, respectively. Clearly,

TABLE III. Characteristics (location and height of the first solvation peak) of liquid phase radial distribution function for argon with different methods and LJ potential.

\begin{tabular}{|c|c|c|}
\hline Functional & $r(\AA)$ & $g(r)$ \\
\hline PBE-D3 & 3.86 & 2.808 \\
\hline BLYP-D3 & 3.74 & 3.174 \\
\hline rVV10 & 3.66 & 3.784 \\
\hline PBE0-D3 & 3.70 & 3.395 \\
\hline M062X-D3 & 3.70 & 2.632 \\
\hline MP2 & 3.66 & 2.982 \\
\hline LJ (Vrabec et al..$\left.^{23}\right)$ & 3.67 & 2.943 \\
\hline Experiment $^{125}$ & 3.68 & 3.050 \\
\hline
\end{tabular}

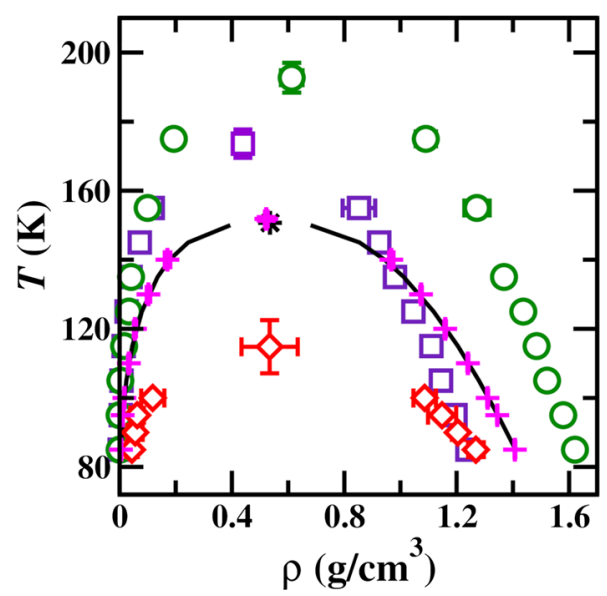

FIG. 5. Vapor-liquid coexistence curves (VLCCs) for argon with different functionals and LJ potential. The solid black lines depict the experimental data and the star represents the experimental critical point. ${ }^{124}$ The red diamonds, violet squares, green circles, and magenta pluses represent GEMC simulation data with BLYP-D3, PBE-D3, rVV10, and LJ (Vrabec et al. ${ }^{23}$ ) potential, respectively. The error bars smaller than the symbol size are not shown.

PBE-D3 functional's performance is superior to the BLYP-D3 functional. Nonetheless, both GGA functionals fail to mimic the slope of the saturated liquid and vapor lines. At higher temperatures, the BLYP-D3 functional gives larger errors as compared to lower temperatures, whereas for the PBE-D3 functional agreement with experimental data improves with the increase in temperature. The results obtained from both these functionals show the underestimation of dispersion interactions. In the case of the rVV10 functional, the overprediction for saturated liquid densities is nearly $23 \%$. The complete curve shows consistent overprediction of the saturated liquid densities and underprediction of the saturated vapor densities. In other words, the dispersion interactions are largely overestimated.

Next, we have used saturated vapor densities to compute the saturated vapor pressures via the ideal gas law. This is an approximation which holds well at lower vapor densities. In Fig. 6, the Clausius-Clapeyron plots compare the saturated vapor pressures obtained from PBE-D3, BLYP-D3, and rVV10 functionals for argon. The BLYP-D3 functional overpredicts the saturated vapor pressures as compared to the experimental data, and this is largely due to the higher vapor densities from the VLCCs. The saturated vapor pressure curves from the PBE-D3 functional show moderate underprediction for all the state points considered in this study. In the case of the rVV10 functional, the saturated vapor pressure curve shows underprediction with larger standard deviation, especially at the lower temperatures. The underprediction of the saturated vapor pressure for PBE-D3 and rVV10 functionals is directly associated with the underprediction of saturated vapor densities.

The subcritical VLE data can be used to estimate the critical properties of any compound. The predicted critical temperature $\left(T_{\mathrm{C}}\right)$, critical densities $\left(\rho_{\mathrm{C}}\right)$, and normal boiling points $\left(T_{\mathrm{B}}\right)$ are presented in Table IV. The critical temperature for argon is overestimated by $15.2 \%$ and $27.8 \%$ with the PBE-D3 and rVV10 functionals, respectively. The high error for the rVV10 functional is due to the overprediction 


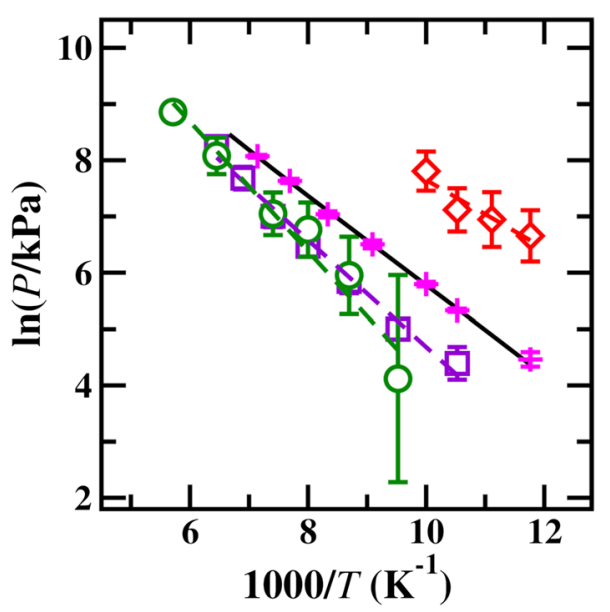

FIG. 6. Clausius-Clapeyron plots for argon with different functionals and LJ potential. The solid black lines depict the experimental data. ${ }^{124}$ The red diamonds, violet squares, green circles, and magenta pluses represent GEMC simulation data with BLYP-D3, PBE-D3, rVV10, and LJ (Vrabec et al. ${ }^{23}$ ) potential, respectively. The error bars smaller than symbol size are not shown. The dashed lines show a linear fit of the simulation data.

of saturated liquid densities and underprediction of saturated vapor densities. For PBE-D3, it still performs reasonably well for saturated liquid densities but underprediction of saturated vapor densities takes the critical temperature to a higher value. The critical temperature for argon with the BLYP-D3 functional is underestimated by $24 \%$, and this is mainly because of the incorrect curvature of the saturated liquid and vapor density curves. The normal boiling point is obtained by using the Clausius-Clapeyron equation. The PBE-D3 and rVV10 functionals overestimate the boiling point by $17 \%$ and $20 \%$, respectively, whereas the BLYP-D3 functional underpredict by $24 \%$. The LJ potential, ${ }^{23}$ on the other hand, accurately predicts these thermodynamic properties, which is not surprising given that these are parameterized to predict bulk thermodynamic properties.

The VLCCs obtained from different functionals can also be directly related to the dimer PECs for argon. As mentioned above, both functionals (BLYP-D3 and PBE-D3) give very similar results for the location of the well depth but the difference for the binding energy is approximately $0.7 \mathrm{~kJ} / \mathrm{mol}$. The underestimation of binding for BLYP-D3 leads to lower saturated liquid densities and higher saturated vapor densities, and this behavior ultimately leads to a lower critical temperature. With the PBE-D3 functional, the prediction for saturated liquid densities at lower reduced temperatures $\left(T_{\mathrm{r}}=T / T_{C}\right)$

TABLE IV. Critical temperature $\left(T_{\mathrm{C}}\right)$, critical density $\left(\rho_{\mathrm{C}}\right)$, and normal boiling point $\left(T_{\mathrm{B}}\right)$ for argon obtained from Monte Carlo simulations. Experimental data are taken from the NIST chemistry webbook. ${ }^{124}$ The numbers in the parenthesis are the standard deviations.

\begin{tabular}{|c|c|c|c|}
\hline Functional & $T_{\mathrm{C}}(\mathrm{K})$ & $\rho_{\mathrm{C}}\left(\mathrm{g} / \mathrm{cm}^{3}\right)$ & $T_{\mathrm{B}}(\mathrm{K})$ \\
\hline PBE-D3 & $173.6(4)$ & $0.439(0.03)$ & $102(2)$ \\
\hline BLYP-D3 & $114.8(8)$ & $0.534(0.10)$ & $67(5)$ \\
\hline rVV10 & $192.7(5)$ & $0.612(0.04)$ & $105(3)$ \\
\hline LJ (Vrabec et al. ${ }^{23}$ ) & $152.0(1)$ & $0.522(0.005)$ & $87(1)$ \\
\hline Experiment $^{124}$ & 150.69 & 0.535 & 87.5 \\
\hline
\end{tabular}

are similar to BLYP-D3, but agreement improves at higher reduced temperatures. This is largely due to the difference in binding energy between the functionals. The higher binding energy prediction with PBE-D3 leads to having a higher critical temperature as compared to the prediction from BLYP-D3. The rVV10 functional does not reproduce the actual VLCC behavior in spite of PEC that matches well with the experiment and CCSD(T)/CBS data for a separation less than $4 \AA$. The overprediction for saturated liquid densities is possibly due to overbinding at larger separations. Therefore, at present, the rVV10 functional is evidently one of the best options to determine accurate geometries for dimer energetics, but it is not sufficiently reliable for computing liquid phase properties of the system. In summary, the PBE functional with the D3 dispersion correction model is a good choice to model vapor liquid equilibria for argon when compared with BLYP-D3 and rVV10 functionals.

\section{CONCLUSIONS}

Vapor liquid equilibria along with the liquid density and liquid microstructure of argon are predicted using first principles Gibbs ensemble Monte Carlo simulations. Among different theoretical models considered in the present work, MP2 performs extremely well for liquid and the structure of first solvation shell albeit at a significantly higher computational cost compared to density functional methods. The rVV10 nonlocal van der Waals functional performs well for PEC and second virial coefficients but overpredicts saturated liquid densities, indicating that many body polarization effects are overestimated. The performance of dispersion corrected hybrid functionals (PBE0-D3 and M06-2X-D3) is not as good as MP2 for the densities and location of the first coordination shell. At last, PBE-D3 performs reasonably well for VLE as compared to BLYP-D3 and rVV10. Overall, our work indicates that the MP2/GPW approach is most suitable for predicting condensed phase properties of systems governed by dispersion interactions.

\section{SUPPLEMENTARY MATERIAL}

See supplementary material for bias potential parameters, energy cutoff for different simulations, plot for density versus Monte Carlo steps, numerical data used for VLE and ClausiusClapeyron plots, dimer PEC, second virial coefficients, and basis sets used for RI-MP2 calculations.

\section{ACKNOWLEDGMENTS}

This work is funded by the National Science Foundation (NSF) under Grant No. CHE-1265872. S.L. and B.S. were supported by EPSRC (Nos. EP/K039296/1 and EP/K038400/1). This research used resources of the National Energy Research Scientific Computing Center, a DOE Office of Science User Facility supported by the Office of Science of the U.S. Department of Energy under Contract No. DE-AC02-05CH11231. This work used the Extreme Science and Engineering Discovery Environment (XSEDE), which is supported by National Science Foundation Grant No. ACI1548562. The authors also acknowledge the Texas Advanced 
Computing Center (TACC) at The University of Texas at Austin for providing HPC resources (Stampede) that have contributed to the research results reported within this paper through XSEDE Allocation No. CHE140141. At last, computational resources at High Performance Computing Collaboratory at Mississippi State University were used for this project.

${ }^{1}$ R. A. Aziz and M. Slaman, "The argon and krypton interatomic potentials revisited," Mol. Phys. 58, 679-697 (1986).

${ }^{2}$ W. L. Jorgensen, D. S. Maxwell, and J. Tirado-Rives, "Development and testing of the opls all-atom force field on conformational energetics and properties of organic liquids," J. Am. Chem. Soc. 118, 11225-11236 (1996).

${ }^{3}$ J. Wang, R. M. Wolf, J. W. Caldwell, P. A. Kollman, and D. A. Case, "Development and testing of a general amber force field," J. Comput. Chem. 25, 1157-1174 (2004).

${ }^{4}$ N. Rai and J. I. Siepmann, "Transferable potentials for phase equilibria. 9. Explicit hydrogen description of benzene and five-membered and sixmembered heterocyclic aromatic compounds," J. Phys. Chem. B 111, 10790-10799 (2007).

${ }^{5}$ N. Rai, J. L. Rafferty, A. Maiti, and J. I. Siepmann, "Prediction of the bubble point pressure for the binary mixture of ethanol and 1, 1, 1, 2, 3, 3, 3-heptafluoropropane from gibbs ensemble Monte Carlo simulations using the trappe force field," Fluid Phase Equilib. 260, 199-211 (2007).

${ }^{6} \mathrm{~K}$. Patkowski and K. Szalewicz, "Argon pair potential at basis set and excitation limits," J. Chem. Phys. 133, 094304 (2010).

${ }^{7}$ K. Patkowski, G. Murdachaew, C.-M. Fou, and K. Szalewicz, "Accurate $a b$ initio potential for argon dimer including highly repulsive region," Mol. Phys. 103, 2031-2045 (2005).

${ }^{8}$ P. Slaviček, R. Kalus, P. Paška, I. Odvárková, P. Hobza, and A. Malijevskỳ, "State-of-the-art correlated ab initio potential energy curves for heavy rare gas dimers: $\mathrm{Ar}_{2}, \mathrm{Kr}_{2}$, and $\mathrm{Xe}_{2}$," J. Chem. Phys. 119, 2102-2119 (2003).

${ }^{9}$ C. Desgranges and J. Delhommelle, "Many-body effects on the thermodynamics of fluids, mixtures, and nanoconfined fluids," J. Chem. Theory Comput. 11, 5401-5414 (2015)

${ }^{10}$ A. Eskandari Nasrabad and R. Laghaei, "Computational studies on thermodynamic properties, effective diameters, and free volume of argon using an ab initio potential," J. Chem. Phys. 125, 084510 (2006).

${ }^{11} \mathrm{M}$. Callsen and I. Hamada, "Assessing the accuracy of the van der waals density functionals for rare-gas and small molecular systems," Phys. Rev. B 91, 195103 (2015).

${ }^{12} \mathrm{G}$. Marcelli and R. J. Sadus, "A link between the two-body and three-body interaction energies of fluids from molecular simulation," J. Chem. Phys. 112, 6382-6385 (2000).

${ }^{13}$ A. Hermann and P. Schwerdtfeger, "Complete basis set limit second-order Møller-Plesset calculations for the fcc lattices of neon, argon, krypton, and xenon," J. Chem. Phys. 131, 244508 (2009).

${ }^{14}$ M. J. Van Vleet, A. J. Misquitta, A. J. Stone, and J. R. Schmidt, "Beyond born-mayer: Improved models for short-range repulsion in ab initio force fields," J. Chem. Theory Comput. 12, 3851-3870 (2016).

${ }^{15}$ M. J. McGrath, J. N. Ghogomu, N. T. Tsona, J. I. Siepmann, B. Chen, I. Napari, and H. Vehkamäki, "Vapor-liquid nucleation of argon: Exploration of various intermolecular potentials," J. Chem. Phys. 133, 084106 (2010).

${ }^{16} \mathrm{~K}$. Tang and J. Toennies, "The van der waals potentials between all the rare gas atoms from He to Rn," J. Chem. Phys. 118, 4976-4983 (2003).

${ }^{17}$ B. Jäger, R. Hellmann, E. Bich, and E. Vogel, "Ab initio pair potential energy curve for the argon atom pair and thermophysical properties of the dilute argon gas. I. Argon-argon interatomic potential and rovibrational spectra," Mol. Phys. 107, 2181-2188 (2009).

${ }^{18}$ J. Rezac and P. Hobza, "Benchmark calculations of interaction energies in noncovalent complexes and their applications," Chem. Rev. 116, 50385071 (2016).

${ }^{19}$ E. Ermakova, J. Solca, H. Huber, and M. Welker, "Argon in condensed phase: Quantitative calculations of structural, thermodynamic, and transport properties from pure theory," J. Chem. Phys. 102, 4942-4951 (1995).

${ }^{20}$ E. K. Goharshadi and M. Abbaspour, "Molecular dynamics simulation of argon, krypton, and xenon using two-body and three-body intermolecular potentials," J. Chem. Theory Comput. 2, 920-926 (2006).
${ }^{21}$ G. Marcelli and R. J. Sadus, "Molecular simulation of the phase behavior of noble gases using accurate two-body and three-body intermolecular potentials," J. Chem. Phys. 111, 1533-1540 (1999).

${ }^{22}$ E. Vogel, B. Jäger, R. Hellmann, and E. Bich, "Ab initio pair potential energy curve for the argon atom pair and thermophysical properties for the dilute argon gas. II. Thermophysical properties for low-density argon," Mol. Phys. 108, 3335-3352 (2010).

${ }^{23}$ J. Vrabec, J. Stoll, and H. Hasse, "A set of molecular models for symmetric quadrupolar fluids,” J. Phys. Chem. B 105, 12126-12133 (2001).

${ }^{24}$ M. Vlasiuk and R. J. Sadus, " $A b$ initio interatomic potentials and the thermodynamic properties of fluids," J. Chem. Phys. 147, 024505 (2017).

${ }^{25} \mathrm{M}$. Vlasiuk and R. J. Sadus, "Predicting vapor-liquid phase equilibria with augmented ab initio interatomic potentials," J. Chem. Phys. 146, 244504 (2017).

${ }^{26}$ P. Hohenberg and W. Kohn, "Inhomogeneous electron gas," Phys. Rev. 136, B864 (1964).

${ }^{27}$ W. Kohn and L. J. Sham, "Self-consistent equations including exchange and correlation effects," Phys. Rev. 140, A1133 (1965).

${ }^{28} \mathrm{~S}$. Grimme, "Density functional theory with London dispersion corrections,” Wiley Interdiscip. Rev.: Comput. Mol. Sci. 1, 211-228 (2011).

${ }^{29}$ J. Klimeš and A. Michaelides, "Perspective: Advances and challenges in treating van der waals dispersion forces in density functional theory," J. Chem. Phys. 137, 120901 (2012).

${ }^{30}$ A. J. Cohen, P. Mori-Sánchez, and W. Yang, "Challenges for density functional theory," Chem. Rev. 112, 289-320 (2011).

${ }^{31}$ J. P. Perdew, A. Ruzsinszky, J. Tao, V. N. Staroverov, G. E. Scuseria, and G. I. Csonka, "Prescription for the design and selection of density functional approximations: More constraint satisfaction with fewer fits," J. Chem. Phys. 123, 062201 (2005).

${ }^{32}$ J. P. Perdew and Y. Wang, "Accurate and simple analytic representation of the electron-gas correlation energy," Phys. Rev. B 45, 13244-13249 (1992).

${ }^{33}$ S. Vosko, L. Wilk, and M. Nusair, "Accurate spin-dependent electron liquid correlation energies for local spin density calculations: A critical analysis," Can. J. Phys. 58, 1200-1211 (1980).

${ }^{34}$ J. P. Perdew, "Density-functional approximation for the correlation energy of the inhomogeneous electron gas," Phys. Rev. B 33, 8822-8824 (1986).

${ }^{35}$ A. D. Becke, "Density-functional exchange-energy approximation with correct asymptotic behavior," Phys. Rev. A 38, 3098 (1988).

${ }^{36}$ C. Lee, W. Yang, and R. G. Parr, "Development of the Colle-Salvetti correlation-energy formula into a functional of the electron density," Phys. Rev. B 37, 785 (1988).

${ }^{37}$ J. P. Perdew, K. Burke, and M. Ernzerhof, "Generalized gradient approximation made simple,” Phys. Rev. Lett. 77, 3865 (1996).

${ }^{38}$ J. P. Perdew, S. Kurth, A. Zupan, and P. Blaha, "Accurate density functional with correct formal properties: A step beyond the generalized gradient approximation," Phys. Rev. Lett. 82, 2544 (1999).

${ }^{39}$ T. Van Voorhis and G. E. Scuseria, "A novel form for the exchangecorrelation energy functional," J. Chem. Phys. 109, 400-410 (1998).

${ }^{40}$ M. Ernzerhof and G. E. Scuseria, "Kinetic energy density dependent approximations to the exchange energy," J. Chem. Phys. 111, 911-915 (1999).

${ }^{41}$ A. D. Becke, "Exploring the limits of gradient corrections in density functional theory," J. Comput. Chem. 20, 63-69 (1999).

${ }^{42}$ Y. Zhao, N. E. Schultz, and D. Truhlar, "Exchange-correlation functional with broad accuracy for metallic and nonmetallic compounds, kinetics, and noncovalent interactions," J. Chem. Phys. 123, 161103 (2005).

${ }^{43}$ A. D. Becke, "Density-functional thermochemistry. III. The role of exact exchange," J. Chem. Phys. 98, 5648-5652 (1993).

${ }^{44}$ P. Stephens, F. Devlin, C. Chabalowski, and M. J. Frisch, "Ab initio calculation of vibrational absorption and circular dichroism spectra using density functional force fields," J. Phys. Chem. 98, 11623-11627 (1994).

${ }^{45} \mathrm{C}$. Adamo and V. Barone, "Toward reliable density functional methods without adjustable parameters: The PBE0 model," J. Chem. Phys. 110, 6158-6170 (1999).

${ }^{46}$ J. Heyd, G. E. Scuseria, and M. Ernzerhof, "Hybrid functionals based on a screened Coulomb potential," J. Chem. Phys. 118, 8207-8215 (2003).

${ }^{47}$ Y. Zhao and D. G. Truhlar, "Density functionals with broad applicability in chemistry," Acc. Chem. Res. 41, 157-167 (2008).

${ }^{48}$ A. Heßelmann and A. Görling, "Random-phase approximation correlation methods for molecules and solids," Mol. Phys. 109, 2473-2500 (2011).

${ }^{49}$ X. Ren, P. Rinke, C. Joas, and M. Scheffler, "Random-phase approximation and its applications in computational chemistry and materials science," J. Mater. Sci. 47, 7447-7471 (2012). 
${ }^{50}$ H. Eshuis, J. E. Bates, and F. Furche, "Electron correlation methods based on the random phase approximation," Theor. Chem. Acc. 131, 1084 (2012).

${ }^{51}$ G. P. Chen, V. K. Voora, M. M. Agee, S. G. Balasubramani, and F. Furche, "Random-phase approximation methods," Annu. Rev. Phys. Chem. 68, 421-445 (2017).

${ }^{52}$ J. Tao, J. P. Perdew, V. N. Staroverov, and G. E. Scuseria, "Climbing the density functional ladder: Nonempirical meta-generalized gradient approximation designed for molecules and solids," Phys. Rev. Lett. 91, 146401 (2003).

${ }^{53}$ J. P. Perdew, "Climbing the ladder of density functional approximations," MRS Bull. 38, 743-750 (2013).

${ }^{54}$ J. P. Perdew and K. Schmidt, "Jacob's ladder of density functional approximations for the exchange-correlation energy," AIP Conf. Proc. 577, 1-20 (2001).

${ }^{55} \mathrm{~S}$. Grimme, "Accurate description of van der Waals complexes by density functional theory including empirical corrections," J. Comput. Chem. 25, 1463-1473 (2004).

${ }^{56}$ S. Grimme, "Semiempirical GGA-type density functional constructed with a long-range dispersion correction," J. Comput. Chem. 27, 1787-1799 (2006).

${ }^{57}$ S. Grimme, J. Antony, S. Ehrlich, and H. Krieg, "A consistent and accurate ab initio parametrization of density functional dispersion correction (DFTD) for the 94 elements H-Pu," J. Chem. Phys. 132, 154104 (2010).

${ }^{58}$ M. Dion, H. Rydberg, E. Schröder, D. C. Langreth, and B. I. Lundqvist, "Van der Waals density functional for general geometries," Phys. Rev. Lett. 92, 246401 (2004).

${ }^{59}$ D. É. Murray, K. Lee, and D. C. Langreth, "Investigation of exchange energy density functional accuracy for interacting molecules," J. Chem. Theory Comput. 5, 2754-2762 (2009).

${ }^{60} \mathrm{O}$. A. Vydrov and T. Van Voorhis, "Nonlocal van der Waals density functional made simple," Phys. Rev. Lett. 103, 063004 (2009).

${ }^{61}$ K. Lee, D. É. Murray, L. Kong, B. I. Lundqvist, and D. C. Langreth, "Higher-accuracy van der waals density functional," Phys. Rev. B 82 , 081101 (2010).

${ }^{62}$ I.-F. W. Kuo, C. J. Mundy, M. J. McGrath, J. I. Siepmann, J. VandeVondele, M. Sprik, J. Hutter, B. Chen, M. L. Klein, F. Mohamed et al., "Liquid water from first principles: Investigation of different sampling approaches," J. Phys. Chem. B 108, 12990-12998 (2004).

${ }^{63}$ M. McGrath, J. Siepmann, I.-F. Kuo, and C. Mundy, "Vapor-liquid equilibria of water from first principles: Comparison of density functionals and basis sets," Mol. Phys. 104, 3619-3626 (2006).

${ }^{64}$ M. J. McGrath, J. I. Siepmann, I.-F. W. Kuo, C. J. Mundy, J. VandeVondele, J. Hutter, F. Mohamed, and M. Krack, "Simulating fluid-phase equilibria of water from first principles," J. Phys. Chem. A 110, 640-646 (2006).

${ }^{65}$ M. J. McGrath, I.-F. W. Kuo, J. N. Ghogomu, C. J. Mundy, and J. I. Siepmann, "Vapor-liquid coexistence curves for methanol and methane using dispersion-corrected density functional theory," J. Phys. Chem. B 115, 11688-11692 (2011)

${ }^{66}$ H. Goel, C. L. Butler, Z. W. Windom, and N. Rai, "Vapor liquid equilibria of hydrofluorocarbons using dispersion-corrected and nonlocal density functionals," J. Chem. Theory Comput. 12, 3295-3304 (2016).

${ }^{67}$ H. Goel, Z. W. Windom, C. L. Butler, and N. Rai, "Phase equilibria and condensed phase properties of fluorinated alkanes via first principles simulations," ChemistrySelect 2, 11969-11976 (2017).

${ }^{68} \mathrm{H}$. Goel, Z. W. Windom, A. A. Jackson, and N. Rai, "Performance of density functionals for modeling vapor liquid equilibria of $\mathrm{CO}_{2}$ and $\mathrm{SO}_{2}$,", J. Comput. Chem. 39, 397-406 (2018).

${ }^{69}$ K. A. Maerzke, G. Murdachaew, C. J. Mundy, G. K. Schenter, and J. I. Siepmann, "Self-consistent polarization density functional theory: Application to argon," J. Phys. Chem. A 113, 2075-2085 (2009).

${ }^{70}$ M. Del Ben, J. Hutter, and J. VandeVondele, "Probing the structural and dynamical properties of liquid water with models including non-local electron correlation," J. Chem. Phys. 143, 054506 (2015).

${ }^{71}$ M. Del Ben, M. Schönherr, J. Hutter, and J. VandeVondele, "Bulk liquid water at ambient temperature and pressure from MP2 theory," J. Phys. Chem. Lett. 4, 3753-3759 (2013).

${ }^{72}$ M. Del Ben, J. VandeVondele, and B. Slater, "Periodic MP2, RPA, and boundary condition assessment of hydrogen ordering in ice XV," J. Phys. Chem. Lett. 5, 4122-4128 (2014).

${ }^{73}$ M. Del Ben, J. Hutter, and J. VandeVondele, "Forces and stress in second order Møller-Plesset perturbation theory for condensed phase systems within the resolution-of-identity Gaussian and plane waves approach," J. Chem. Phys. 143, 102803 (2015)
${ }^{74}$ T. Todorova, A. P. Seitsonen, J. Hutter, I.-F. W. Kuo, and C. J. Mundy, "Molecular dynamics simulation of liquid water: Hybrid density functionals," J. Phys. Chem. B 110, 3685-3691 (2006).

${ }^{75}$ M. Guidon, F. Schiffmann, J. Hutter, and J. VandeVondele, "A $b$ initio molecular dynamics using hybrid density functionals," J. Chem. Phys. 128 214104 (2008)

${ }^{76} \mathrm{M}$. Guidon, J. Hutter, and J. VandeVondele, "Auxiliary density matrix methods for Hartree-Fock exchange calculations," J. Chem. Theory Comput. 6 , 2348-2364 (2010).

${ }^{77}$ R. A. DiStasio, Jr., B. Santra, Z. Li, X. Wu, and R. Car, "The individual and collective effects of exact exchange and dispersion interactions on the $a b$ initio structure of liquid water," J. Chem. Phys. 141, 084502 (2014).

${ }^{78}$ C. Zhang, D. Donadio, F. Gygi, and G. Galli, "First principles simulations of the infrared spectrum of liquid water using hybrid density functionals," J. Chem. Theory Comput. 7, 1443-1449 (2011).

${ }^{79}$ M. Schönherr, B. Slater, J. Hutter, and J. VandeVondele, "Dielectric properties of water ice, the ice $\mathrm{Ih} / \mathrm{XI}$ phase transition, and an assessment of density functional theory," J. Phys. Chem. B 118, 590-596 (2014).

${ }^{80}$ Q. Wan, L. Spanu, F. Gygi, and G. Galli, "Electronic structure of aqueous sulfuric acid from first-principles simulations with hybrid functionals," J. Phys. Chem. Lett. 5, 2562-2567 (2014).

${ }^{81}$ M. Macher, J. Klimeš, C. Franchini, and G. Kresse, "The random phase approximation applied to ice," J. Chem. Phys. 140, 084502 (2014).

${ }^{82}$ A. P. Gaiduk, F. Gygi, and G. Galli, "Density and compressibility of liquid water and ice from first-principles simulations with hybrid functionals," J. Phys. Chem. Lett. 6, 2902-2908 (2015).

${ }^{83}$ F. Ambrosio, G. Miceli, and A. Pasquarello, "Structural, dynamical, and electronic properties of liquid water: A hybrid functional study," J. Phys. Chem. B 120, 7456-7470 (2016).

${ }^{84}$ M. J. Gillan, D. Alfè, and A. Michaelides, "Perspective: How good is DFT for water?," J. Chem. Phys. 144, 130901 (2016).

${ }^{85}$ S. Velasco, F. L. Román, and J. A. White, "On the Clausius-Clapeyron vapor pressure equation," J. Chem. Educ. 86, 106 (2009).

${ }^{86}$ R. Sabatini, T. Gorni, and S. de Gironcoli, "Nonlocal van der Waals density functional made simple and efficient," Phys. Rev. B 87, 041108 (2013).

${ }^{87}$ O. A. Vydrov and T. Van Voorhis, "Nonlocal van der Waals density functional: The simpler the better," J. Chem. Phys. 133, 244103 (2010).

${ }^{88} \mathrm{~F}$. Tran and J. Hutter, "Nonlocal van der Waals functionals: The case of rare-gas dimers and solids," J. Chem. Phys. 138, 204103 (2013).

${ }^{89}$ M. Ernzerhof and G. E. Scuseria, "Assessment of the Perdew-BurkeErnzerhof exchange-correlation functional," J. Chem. Phys. 110, 50295036 (1999).

${ }^{90} \mathrm{C}$. Møller and M. S. Plesset, "Note on an approximation treatment for many-electron systems," Phys. Rev. 46, 618 (1934).

${ }^{91}$ R. J. Bartlett, I. Grabowski, S. Hirata, and S. Ivanov, "The exchangecorrelation potential in ab initio density functional theory," J. Chem. Phys. 122, 034104 (2005)

${ }^{92} \mathrm{~T}$. Schwabe and S. Grimme, "Towards chemical accuracy for the thermodynamics of large molecules: New hybrid density functionals including non-local correlation effects," Phys. Chem. Chem. Phys. 8, 4398-4401 (2006).

${ }^{93} \mathrm{~S}$. Grimme, "Semiempirical hybrid density functional with perturbative second-order correlation," J. Chem. Phys. 124, 034108 (2006).

${ }^{94}$ L. Goerigk and S. Grimme, "Efficient and accurate double-hybridmeta-GGA density functionals-evaluation with the extended GMTKN30 database for general main group thermochemistry, kinetics, and noncovalent interactions," J. Chem. Theory Comput. 7, 291-309 (2010).

${ }^{95}$ A. Z. Panagiotopoulos, "Direct determination of phase coexistence properties of fluids by Monte Carlo simulation in a new ensemble," Mol. Phys. 61, 813-826 (1987)

${ }^{96}$ I. McDonald, "NpT-ensemble Monte Carlo calculations for binary liquid mixtures," Mol. Phys. 23, 41-58 (1972).

${ }^{97}$ D. Frenkel and B. Smit, Understanding Molecular Simulation: From Algorithms to Applications (Academic Press, 2001), Vol. 1, pp. 201-223.

${ }^{98}$ S. Goedecker, M. Teter, and J. Hutter, "Separable dual-space Gaussian pseudopotentials," Phys. Rev. B 54, 1703 (1996).

${ }^{99} \mathrm{C}$. Hartwigsen, S. Gædecker, and J. Hutter, "Relativistic separable dualspace Gaussian pseudopotentials from H to Rn," Phys. Rev. B 58, 3641 (1998).

${ }^{100}$ M. Del Ben, J. Hutter, and J. VandeVondele, "Second-order Møller-Plesset perturbation theory in the condensed phase: An efficient and massively parallel Gaussian and plane waves approach," J. Chem. Theory Comput. 8, 4177-4188 (2012). 
${ }^{101}$ M. Del Ben, J. Hutter, and J. VandeVondele, "Electron correlation in the condensed phase from a resolution of identity approach based on the Gaussian and plane waves scheme," J. Chem. Theory Comput. 9, 2654-2671 (2013).

${ }^{102}$ J. Hutter, M. Iannuzzi, F. Schiffmann, and J. VandeVondele, “Cp2k: Atomistic simulations of condensed matter systems," Wiley Interdiscip. Rev.: Comput. Mol. Sci. 4, 15-25 (2014).

${ }^{103}$ J. VandeVondele, M. Krack, F. Mohamed, M. Parrinello, T. Chassaing, and J. Hutter, "Quickstep: Fast and accurate density functional calculations using a mixed Gaussian and plane waves approach," Comput. Phys. Commun. 167, 103-128 (2005).

${ }^{104}$ R. Iftimie, D. Salahub, D. Wei, and J. Schofield, "Using a classical potential as an efficient importance function for sampling from an $a b$ initio potential," J. Chem. Phys. 113, 4852-4862 (2000).

${ }^{105}$ L. D. Gelb, "Monte Carlo simulations using sampling from an approximate potential," J. Chem. Phys. 118, 7747-7750 (2003).

${ }^{106}$ J. I. Siepmann and D. Frenkel, "Configurational bias Monte Carlo: A new sampling scheme for flexible chains,” Mol. Phys. 75, 59-70 (1992).

${ }^{107}$ B. Smit, S. Karaborni, and J. I. Siepmann, "Computer simulations of vaporliquid phase equilibria of n-alkanes,” J. Chem. Phys. 102, 2126-2140 (1995).

${ }^{108}$ A. Michels, H. Wijker, and H. Wijker, "Isotherms of argon between $0{ }^{\circ} \mathrm{C}$ and $150{ }^{\circ} \mathrm{C}$ and pressures up to 2900 atmospheres," Physica 15, 627-633 (1949).

${ }^{109}$ B. Smit, P. De Smedt, and D. Frenkel, "Computer simulations in the Gibbs ensemble," Mol. Phys. 68, 931-950 (1989).

${ }^{110}$ J. S. Rowlinson and B. Widom, Molecular Theory of Capillarity (Oxford University Press, New York, 1989), p. 261.

${ }^{111}$ J. S. Rowlinson and F. L. Swinton, Liquids and Liquid Mixtures (Butterworth, London, 1982), pp. 59-85.

${ }^{112}$ F. Coester, "Bound states of a many-particle system," Nucl. Phys. 7, 421-424 (1958)

${ }^{113}$ F. Coester and H. Kümmel, "Short-range correlations in nuclear wave functions," Nucl. Phys. 17, 477-485 (1960).

${ }^{114}$ J. Č́́žek, "On the correlation problem in atomic and molecular systems. Calculation of wavefunction components in ursell-type expansion using quantum-field theoretical methods," J. Chem. Phys. 45, 4256-4266 (1966).

${ }^{115}$ J. Čížek, "On the use of the cluster expansion and the technique of diagrams in calculations of correlation effects in atoms and molecules," Adv. Chem. Phys. 14, 35 (1969).

${ }^{116}$ J. Č́ížek and J. Paldus, "Correlation problems in atomic and molecular systems III. Rederivation of the coupled-pair many-electron theory using the traditional quantum chemical methodst," Int. J. Quantum Chem. 5, 359-379 (1971).

${ }^{117}$ K. Raghavachari, G. W. Trucks, J. A. Pople, and M. Head-Gordon, "A fifthorder perturbation comparison of electron correlation theories," Chem. Phys. Lett. 157, 479-483 (1989).

${ }^{118}$ T. H. Dunning, Jr., "Gaussian basis sets for use in correlated molecular calculations. I. The atoms boron through neon and hydrogen," J. Chem. Phys. 90, 1007-1023 (1989).

${ }^{119}$ R. A. Kendall, T. H. Dunning, Jr., and R. J. Harrison, "Electron affinities of the first-row atoms revisited. Systematic basis sets and wave functions," J. Chem. Phys. 96, 6796-6806 (1992).

${ }^{120}$ S. F. Boys and F. Bernardi, "The calculation of small molecular interactions by the differences of separate total energies. Some procedures with reduced errors," Mol. Phys. 19, 553-566 (1970).

${ }^{121}$ M. J. Frisch, G. W. Trucks, H. B. Schlegel, G. E. Scuseria, M. A. Robb, J. R. Cheeseman, G. Scalmani, V. Barone, B. Mennucci, G. A. Petersson, H. Nakatsuji, M. Caricato, X. Li, H. P. Hratchian, A. F. Izmaylov, J. Bloino, G. Zheng, J. L. Sonnenberg, M. Hada, M. Ehara, K. Toyota, R. Fukuda, J. Hasegawa, M. Ishida, T. Nakajima, Y. Honda, O. Kitao, H. Nakai, T. Vreven, J. A. Montgomery, Jr., J. E. Peralta, F. Ogliaro, M. Bearpark, J. J. Heyd, E. Brothers, K. N. Kudin, V. N. Staroverov, R. Kobayashi, J. Normand, K. Raghavachari, A. Rendell, J. C. Burant, S. S. Iyengar, J. Tomasi, M. Cossi, N. Rega, J. M. Millam, M. Klene, J. E. Knox, J. B. Cross, V. Bakken, C. Adamo, J. Jaramillo, R. Gomperts, R. E. Stratmann, O. Yazyev, A. J. Austin, R. Cammi, C. Pomelli, J. W. Ochterski, R. L. Martin, K. Morokuma, V. G. Zakrzewski, G. A. Voth, P. Salvador, J. J. Dannenberg, S. Dapprich, A. D. Daniels, A. Farkas, J. B. Foresman, J. V. Ortiz, J. Cioslowski, and D. J. Fox, Gaussian 09, Revision C.1, Gaussian, Inc., Wallingford, CT, 2009.

${ }^{122}$ P. Herman, P. LaRocque, and B. Stoicheff, "Vacuum ultraviolet laser spectroscopy. V. Rovibronic spectra of $\mathrm{Ar}_{2}$ and constants of the ground and excited states," J. Chem. Phys. 89, 4535-4549 (1988).

${ }^{123}$ J. H. Dymond and E. B. Smith, Virial Coefficients of Pure Gases and Mixtures. A Critical Compilation (Oxford University Press, Fair Lawn, NJ, 1980).

${ }^{124}$ NIST Chemistry WebBook, NIST Standard Reference Database Number 69, edited by P. J. Linstrom and W. G. Mallard (National Institute of Standards and Technology, Gaithersburg, MD, 2017), Available at http://webbook.nist.-gov., accessed: 05-05-2017.

${ }^{125}$ J. Yarnell, M. Katz, R. G. Wenzel, and S. Koenig, "Structure factor and radial distribution function for liquid argon at 85 k," Phys. Rev. A 7, 2130 (1973). 